\title{
ON A PROPERTY OF $k$ CONSECUTIVE INTEGERS ${ }^{1}$
}

\section{ALFRED BRAUER}

S. S. Pillai ${ }^{2}$ has just proved the following theorem: In every set of less than 17 consecutive integers there exists at least one integer which is relatively prime to all the others; there are sequences of $k$ integers for $k=17,18, \cdots, 430$, however, which have not this property. Pillai conjectures that the same is valid for every $k \geqq 17$. I shall prove that this conjecture is true.

The method of the proof is similar to the method I applied in a joint paper with $\mathrm{H}$. Zeitz ${ }^{3}$ in proving that the following conjecture is wrong for every prime $p \geqq 43$.

Denote by $p_{n}$ the nth prime. Then there exist at most $2 p_{n-1}-1$ consecutive integers such that each of these integers is at least divisible by one of the primes $p_{1}, p_{2}, \cdots, p_{n}$.

This conjecture was used by Legendre for his proof of the theorem of the primes in arithmetical progressions. First I prove the following.

Lemma. Let $\pi(x)$ be the number of primes $p \leqq x$. Then we have

$$
\pi(2 x)-\pi(x) \geqq 2\left[\frac{\log x}{\log 2}\right]+2
$$

for every $x \geqq 75$.

Proof. If we put, as usual,

$$
\vartheta(x)=\sum_{p \leqq x} \log p,
$$

then we have

$$
\begin{aligned}
\pi(2 x)-\pi(x) & =\sum_{x<p \leqq 2 x} 1 \geqq \sum_{x<p \leqq 2 x}(\log p / \log 2 x) \\
& =\left\{\sum_{x<p \leqq 2 x} \log p\right\} / \log 2 x=\{\vartheta(2 x)-\vartheta(x)\} / \log 2 x .
\end{aligned}
$$

${ }^{1}$ Presented to the Society, September 12, 1940.

${ }^{2}$ S. S. Pillai, On $m$ consecutive integers, Proceedings of the Indian Academy of Sciences, section A, vol. 11 (1940), pp. 6-12.

${ }^{3} \mathrm{~A}$. Brauer und H. Zeitz, Über eine zahlentheoretische Behauptung von Legendre, Sitzungsberichte der Berliner mathematischen Gesellschaft, vol. 29 (1930), pp. 116125. Cf. A. Brauer, Question concerning the maximum term in the diatomic seriesproposed by A. A. Bennett, American Mathematical Monthly, vol. 40 (1933), pp. 409410 . 
It is known that ${ }^{4}$

$$
\begin{aligned}
\vartheta(2 x)-\vartheta(x) \geqq & \vartheta(2 x-2)-\vartheta(x)>.7 x-3.4 x^{1 / 2} \\
& -4.5 \log ^{2} x-24 \log x-32 .
\end{aligned}
$$

Hence, by (1), (2), and (3), it is sufficient to prove that $.7 x-3.4 x^{1 / 2}-4.5 \log ^{2} x-24 \log x-32>(2 \log x / \log 2+2)(\log x+\log 2)$, $f(x)=.7 x-3.4 x^{1 / 2}-\log ^{2} x(4.5+2 / \log 2)-28 \log x-32-2 \log 2>0$.

It is easy to see that $f(x)>0$ holds for $x=1024$, since $\log 1024<7$. Moreover we have

$f^{\prime}(x)=.7-1.7 x^{-1 / 2}-\frac{9+4 / \log 2}{x} \log x-\frac{28}{x}>0 \quad$ for $x \geqq 1024$.

Hence $f(x)$ is increasing for $x \geqq 1024$ and the lemma is proved for $x \geqq 1024$.

For $75 \leqq x<1024$ the lemma can be proved directly. For instance, it follows for $591 \leqq x<1024$ and for $355 \leqq x<591$ by the fact that there are 22 primes between 1024 and 1182 and 20 primes between 591 and 710 . In the same way we get the lemma for $231 \leqq x<355$, $159 \leqq x<231$, and so on.

THEOREM. For every $k \geqq 17$ there exists a sequence of $k$ consecutive integers such that none of these $k$ integers is relatively prime to the product of the others.

Proof. In view of the paper of Pillai, it is sufficient to prove the theorem for $k \geqq 300$. We put

$$
m=\left[\frac{k}{4}\right] \geqq 75 .
$$

Let $p_{1}, p_{2}, \cdots, p_{r}$ be the primes in the closed interval $\{1 \cdots m\}$ and $p_{r+1}, p_{r+2}, \cdots, p_{s}$ the primes in the closed interval $\{m+1 \cdots 2 m\}$. If we consider $k$ consecutive integers, then each of the primes

$$
p_{1}, p_{2}, \cdots, p_{r}, p_{r+1}, p_{r+2}, \cdots, p_{s}
$$

divides at least two of the $k$ integers, since each of these primes is less than $2 m$, hence by (4) less than $k / 2$. Therefore each of these $k$ integers which is divisible by at least one of the primes (5) is not relatively prime to all the $k-1$ other integers. Hence it is sufficient to

\footnotetext{
${ }^{4}$ See, for example, E. Landau, Handbuch der Lehre von der Verteilung der Primzahlen, vol. 1, 1909, p. 91.
} 
prove that there exist sequences of $k$ integers such that for $k \geqq 300$ each of these integers is divisible by at least one of the primes (5).

We consider the simultaneous congruences

$$
x \equiv 1(\bmod 2), \quad x \equiv 0\left(\bmod p_{2} p_{3} \cdots p_{r}\right) .
$$

Let $x$ be a solution of (6). Then the integers

$$
x-2 m, x-2 m+2, \cdots, x-2, x, x+2, \cdots, x+2 m-2, x+2 m
$$

form a sequence of $2 m+1$ odd integers of the form

$$
x \pm 2 \mu, \quad \mu=0,1, \cdots, m .
$$

If $\mu$ is divisible by the odd prime $p_{\nu}$, we have $p_{\nu} \leqq p_{r}$, since $\mu \leqq m$ because of (8). Hence we obtain from (6) that

$$
x \pm 2 \mu \equiv 0\left(\bmod p_{v}\right) .
$$

It follows from (9) that all those integers of (7) which have not the form $x \pm 2^{r}$ with $\tau \geqq 1$ are divisible by at least one of the primes $p_{2}, p_{3}, \cdots, p_{r}$.

If we put

$$
\left[\frac{\log m}{\log 2}\right]+1=t
$$

then the integers of the form $x \pm 2^{\tau}$ with $\tau \geqq 1$ in the set (7) are the integers

$$
x \pm 2, x \pm 2^{2}, \cdots, x \pm 2^{t} .
$$

By (4), it follows from the lemma and from (10) that the number of primes in the closed interval $\{m+1 \cdots 2 m\}$ is

$$
\pi(2 m)-\pi(m) \geqq 2\left[\frac{\log m}{\log 2}\right]+2=2 t .
$$

On the other hand the primes in this interval were $p_{r+1}, p_{r+2}, \cdots, p_{s}$, hence

$$
s-r \geqq 2 t, \quad p_{r+2 t} \leqq p_{s} .
$$

Beside the congruences (6) we now subject $x$ to the following $2 t$ congruences

$$
\begin{aligned}
x+2^{\tau} & \equiv 0\left(\bmod p_{r+\tau}\right), \\
x-2^{\tau} & \equiv 0\left(\bmod p_{r+t+\tau}\right),
\end{aligned} \quad \tau=1,2, \cdots, t .
$$

These congruences always have solutions. For every solution $x$ all the 
numbers (7) are divisible by at least one of the primes (5), since each of the integers (11) is divisible by at least one of the primes $p_{r+1}, p_{r+2}, \cdots, p_{s}$ because of (13) and (12).

Hence each of the $4 m+3$ consecutive integers

(14) $x-2 m-1, x-2 m, x-2 m+1, \cdots, x-1, x, x+1, \cdots, x+2 m+1$ is divisible by at least one of the primes (5), since

$$
\begin{aligned}
x-2 m-1 & \equiv x-2 m+1 \equiv \cdots \equiv x-1 \equiv x+1 \\
& \equiv \cdots \equiv x+2 m+1 \equiv 0(\bmod 2) .
\end{aligned}
$$

Because of (4) we have

$$
k \leqq 4 m+3 .
$$

Therefore we can take $k$ consecutive integers from (14). None of these $k$ integers is relatively prime to the product of the $k-1$ others.

Institute For AdVANCEd Study 